Military Technical College

Kobry El-Kobbah,

Cairo, Egypt

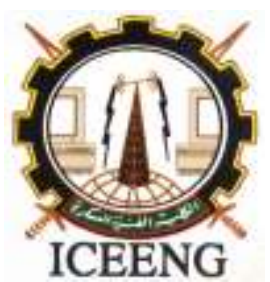

\section{1th International Conference \\ on Electrical Engineering \\ ICEENG 2018}

\title{
New SAR Algorithm for Sidelobe Reduction in Range direction
}

\author{
Ahmed Azouz ${ }^{*}$ Ahmed Eldemiry* and Abdelhamid Gaafar ${ }^{* *}$
}

\begin{abstract}
:
Sidelobes degrade radar performance by placing energy up and down range from its source. Range sidelobes associated with a large radar cross section (RCS) target may mask the presence of a smaller target located within a pulse width of the larger target; thus, "low" sidelobes are a desirable property. All the synthetic aperture radar (SAR) Sidelobe reduction algorithms which using matched filtering (windowing) like hamming window reduce the sidelobes on the expense of degrading the resolution. Our proposed algorithm using an optimum filter is reduced the sidelobes in range direction while keeping range resolution. In this paper, drive the formula for filter that reduce the sidelobe after linear frequency modulation matched filter then check the ambiguity function for proposed filter. Finally applied simulated and real raw data of SAR to proposal algorithm for SAR image formation and range Doppler algorithm and measure the quality of focused image.
\end{abstract}

\section{Keywords:}

SAR, linear frequency modulation (LFM), Optimum filter, Range resolution, Sidelobe reduction.

\section{Introduction}

The signal output when the patch is being imaged is the sum of the simultaneously received power of the range sidelobes and the azimuth sidelobes plus the receiver noise to the extent that this power is comparable to that received from the surrounding terrain.

* Egyptian Armed Forces.

** Communication Dep., AASTMT, Cairo, Egypt. 
If nothing is done to reduce the sidelobe, the integrated sidelobe return alone may contain up to 10 percent as much power as the mainlobe return. Consequently, the loss of contrast can be considerable. Like the sidelobes of a real array, the sidelobes of a synthetic array are produced by the elements at the ends of the array. Consequently, just as the sidelobes of a real antenna may be reduced through illumination tapering, the sidelobes of the synthetic array can be reduced by weighting the returns received by the individual array elements (Hamming window) so as to de-emphasize the returns received by the end elements relative to the returns received by the central elements. The cost of this reduction, of course, is a slight loss of resolution $[1,2]$.

The weighting can be conveniently accomplished when the focusing corrections are applied to the stored returns. The loss in resolution is the price of sidelobe reduction and can be avoided by using an optimum filter for sidelobe reduction to lower the sidelobe level than its level with traditional matched filter so the small targets which was masked by the sidelobes of the larger targets will appear in the focused SAR image [3].

All the traditional algorithms use windowing for reducing the side lobe level up to (40) $d B$ which affects the image resolution due to widening of the mainlobe which decrease the sharpness decreasing the resolution [1]. From the point of view of signal processing paradigm, there are two steps of SAR imaging are Data acquisition and Image formation. Data acquisition is the Measurement of the response of the scene of interest over range of frequencies and angles. the resolution obtainable in the final image depends on the frequency and angle parameters. this step is the transformation of the scene from object space to data space. Image formation: matched filtering of the measured data to the predicted responses from the scene of interest. This step is the transformation from measured data to the final synthetic image [2-4]. Image formation can be performed with any of a large number of algorithms, all of them exact implementations of, or approximations to, filtering matched to point target response.

The main SAR processing algorithms are Range Doppler algorithm, Chirp Scaling algorithm, Omega K algorithm, and, SPECAN algorithm [2].

Range Doppler Algorithm Developed in 1976-1978 for processing SEASAT SAR data still widespread till today. Designed to achieve block processing efficiency using frequency domain operations in both range and azimuth while maintain the simplicity of one-dimension operation. It takes advantage of approximate separation of processing in these two directions allowed by large difference in time scale between both of them, also range cell migration correction (RCMC) used between two one dimensional operations. Block processing efficiency also achieved for RCMC operation because it done in both range time and azimuth frequency domain. This domain is called "range Doppler" domain since azimuth frequency is related to Doppler frequency. Algorithm is called Range Doppler Algorithm "RDA" Because RCMC is done in this domain. It has a modification in 1984 by JPL called secondary range compression (SRC) data with moderate amount of squint .When applied to range compression (RC) matched filter SRC compensates range and azimuth coupling of target's phase history removing phase distortions due to squinting. Also it has concept of multi look processing by selecting parts of data spectrum are processed independently then summed incoherently reducing "speckle noise". This reduce SAR resolution but at same time better 
image interpolation [2,3]. Range Doppler Algorithm limitations are: first one is the High computing load is experienced when obtaining high accuracy in RCMC operation, second one is the Hard incorporation azimuth frequency dependence on SRC which can limits its accuracy in certain high squint and wide aperture cases [2].

In this paper we introduce a new matched filter imaging algorithm with a new way of filtering during processing the real raw data that reduce sidelobes levels in range for any linear frequency modulation (LFM) radar systems without the use of any additional weighting or windowing functions as needed in conventional ones also without affecting image resolution. The rest of this paper is organized as follows: In section 2 introduces matched filter response and sidelobe level reduction by windowing. In section 3 presents the optimum filter structure. In section 4 is the steps of the proposed algorithm with matlab simulation and results comparing usage of range Doppler algorithm and our proposed algorithm. In section 5 discussing the ambiguity function in two cases the LFM matched filtering and optimum filter. To verify our proposed algorithm an implementation of the proposed algorithm on real raw data with comparing the resulted image in both cases of range Doppler and proposed algorithms in section 6. Finally, some concluding remarks about the proposed algorithm results and future work are given in Section 7.

\section{Matched Filter Response}

The transmitted LFM waveform of a single amplitude modulated rectangular pulse can be described as [4]:

$$
x(t)=A \operatorname{rect}\left(\frac{t}{T}\right) \exp \left[j\left(2 \pi f_{o} t+\pi K t^{2}\right)\right]
$$

where $A$ is the signal amplitude, $t$ is the time, $T$ is the pulse width, $f_{0}$ is the carrier frequency, $\mathrm{K}$ is the LFM slope, while the rect ( ) function is defined as:

$$
\operatorname{rect}(x)= \begin{cases}1 & |x|<1 / 2 \\ 0 & |x|>1 / 2\end{cases}
$$

LFM slope is specified by $= \pm B / T$, the positive sign indicates up LFM slope (up chirp) while negative sign indicates a down LFM slope (down chirp), and $B$ is the chirp bandwidth. The amplitude modulation is $A_{T}(\mathrm{t})=A \operatorname{rect}(t / T)$ and the phase modulation is represented as a quadratic function of time as [9]:

$$
\phi(t)=\pi K t^{2}
$$

Frequency modulation, defined as the immediate frequency deviation from the carrier frequency $\left(f_{o}\right)$ is expressed in terms of the phase by:

$$
f_{i}(t)=\frac{1}{2 \pi} \frac{d \phi(\mathrm{t})}{d t}
$$

Frequency modulation for LFM waveform is linear with slope equal to $(\alpha)$ 


$$
f_{i}(t)=K t= \pm\left(\frac{B}{T}\right) t, \quad|t| \leq T / 2
$$

The received signal is reflected back to the radar. The echoed signal from target can be expressed as:

$$
u(t)=A_{r} \operatorname{rect}\left(\frac{(t-\tau)}{T}\right) \exp \left[j\left(2 \pi f_{o}(t-\tau)+\pi K(t-\tau)^{2}\right)\right]
$$

where: $A_{r}$ is an attenuated version of $A, \tau$ is the two-way time of flight to the target at range $R$. Then $\tau$ can be obtain as follows:

$$
\tau=\frac{2 R}{c}
$$

where c light speed in atmosphere. Applied matched filter response in the frequency domain is shown in Figure 1.

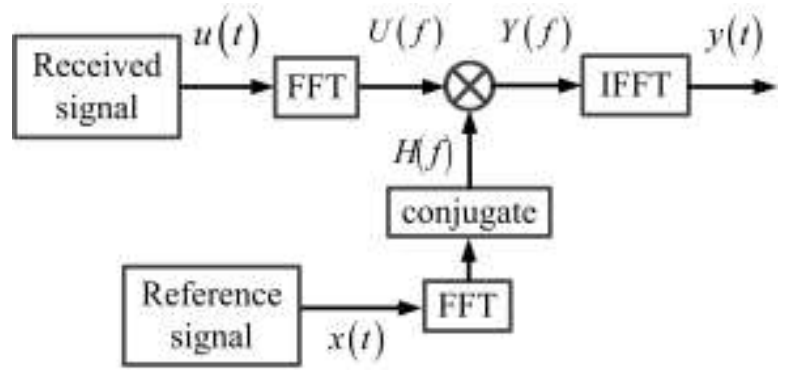

Figure 1. Matched filter components in the frequency domain

Matched filter impulse response is described by [10]

$$
H(f)=(F F T[x(t)])^{*}=A_{H} \operatorname{rect}\left[\frac{\left(f-f_{o}\right)}{|K| T}\right] \cdot \exp \left(+j \pi \frac{\left(f-f_{o}\right)^{2}}{K}\right)
$$

where FFT is the fast Fourier transform, ${ }^{*}$ is the conjugate, and $A_{H}$ is the amplitude of matched filter impulse response.

$$
U(f)=F F T[u(t)]=A_{r}^{\prime} \operatorname{rect}\left[\frac{\left(f-f_{o}\right)}{|K| T}\right] \cdot \exp \left(-j \pi \frac{\left(f-f_{o}\right)^{2}}{K}\right) \cdot \exp (-j 2 \pi f \tau)
$$

where $A_{r}^{\prime}$ is the amplitude of matched filter impulse response. Spectrum of the matched filter output after pulse compression is given by:

$$
Y(f)=F F T[u(t)] \cdot H(f)=A_{Y} \operatorname{rect}\left[\frac{\left(f-f_{o}\right)}{|K| T}\right] \exp (-j 2 \pi f \tau)
$$

where $A_{Y}$ is the amplitude of spectrum of the matched filter output after pulse compression. The compressed signal in time domain is given by inverse Fourier transform (IFFT) of $Y(f)$. 


$$
y(t)=\operatorname{IFFT}[Y(f)]=A_{Y}|K| T \operatorname{sinc}(K T(\mathrm{t}-\tau))
$$

A window is used after matching filtering process to eliminate the impact level of sidelobes generated at the filter output. There are many windowing techniques used in sidelobe elimination or reduction such as Hamming, Hanning, Flattop, and Blackman windowing filters. It has been shown that Hamming windowing has a better performance among other mentioned filter techniques put on the expense of resolution and peak of sidelobe level [5-8].

\section{Optimum Filter derivation}

Our previous consideration of matched filter was operating in continuous domain; to derive the formula of the optimum filter we need to convert our signal to discrete form. The general form in digital form of a single-pulse LFM signal in (10) can be expressed as:

$$
s(n)=A \exp \left(j\left[\omega_{o} n+k \pi n^{2}\right]\right) \quad 0 \leq n \leq N-1
$$

where $A$ is the amplitude, $f_{s}$ is the sampling frequency, $\omega_{0}$ is the angular frequency, $k=$ $B /\left(N f_{s}\right)$ which is LFM coefficient, and $N$ is number of samples. We have two scenarios for optimum filter construction according to the value of the integer $N$, odd or even. Matched filter autocorrelation function is expressed by:

$$
x_{N}(l)=\sum_{n=-\infty}^{\infty} s_{N}^{*}(n) s_{N}(n+l) \quad 0 \leq n \leq N-1
$$

where the asterisk $\left({ }^{*}\right)$ indicates complex conjugate.

\subsection{Odd number samples}

Discrete Fourier Transform (DFT) of auto correlated signal for odd number of samples (let $N=5$ ) can be expressed as:

$$
X_{5}(\omega)=\sum_{l=0}^{M-1} x_{5}(l) e^{-j \frac{2 \pi l \omega}{M}} \quad \text { where } \quad M=9
$$

Output response in time domain, $y(n)$, for $N=5$ samples is set as:

$$
y_{5}(l)=\{0,0,0,0,5,0,0,0,0\}
$$

Spectrum of the output response of (16) can be rewritten as:

$$
Y_{5}(\omega)=5 e^{-j 4 \omega}
$$

The proposed optimum filter transfer function for 5 samples and its simplified form in frequency can be fulfilled as in (17) and (18) respectively. 


$$
H_{5}(\omega)=\frac{Y_{5}(\omega)}{X_{5}(\omega)}=\frac{5}{\left\{\begin{array}{c}
5+2 \cos \left(4\left(\omega-\omega_{o}\right)\right)+2 \cos \left(3\left(k \pi+\omega-\omega_{o}\right)\right)+ \\
2 \cos \left(3\left(k \pi-\omega+\omega_{o}\right)\right)+2 \cos \left(2\left(2 k \pi+\omega-\omega_{o}\right)\right)+ \\
2 \cos \left(2\left(2 k \pi-\omega+\omega_{o}\right)\right)+2 \cos \left(2\left(\omega-\omega_{o}\right)\right)+2 \cos \left(k \pi+\omega-\omega_{o}\right)+ \\
2 \cos \left(k \pi-\omega+\omega_{o}\right)+2 \cos \left(3 k \pi+\omega-\omega_{o}\right)+2 \cos \left(3 k \pi-\omega+\omega_{o}\right)
\end{array}\right\}}
$$

Simplifying (17) we get:

$$
H_{5}(\omega)=\frac{5}{\left\{\begin{array}{c}
5+4[\cos (k \pi)+\cos (3 k \pi)] \cos \left(\omega-\omega_{o}\right)+ \\
2[1+2 \cos (4 k \pi)] \cos \left(2\left(\omega-\omega_{o}\right)\right)+ \\
4 \cos (3 k \pi) \cos \left(3\left(\omega-\omega_{o}\right)\right)
\end{array}\right\}}
$$

Similarly, the proposed optimum filter transfer function for 7 samples can be simplified as:

$$
H_{7}(\omega)=\frac{7}{\left\{\begin{array}{c}
7+4[\cos (k \pi)+\cos (3 k \pi)+\cos (5 k \pi)] \cos \left(\omega-\omega_{o}\right)+2 \cos \left(6\left(\omega-\omega_{o}\right)\right) \\
2[1+2 \cos (4 k \pi)+2 \cos (8 k \pi)] \cos \left(2\left(\omega-\omega_{o}\right)\right)+ \\
4[\cos (3 k \pi)+\cos (9 k \pi)] \cos \left(3\left(\omega-\omega_{o}\right)\right)+ \\
2[1+2 \cos (8 k \pi)] \cos \left(4\left(\omega-\omega_{o}\right)\right)+4 \cos (5 k \pi) \cos \left(5\left(\omega-\omega_{o}\right)\right)
\end{array}\right\}}
$$

Hence, the proposed optimum filter transfer function in its general form for $(M)$ odd samples is written as:

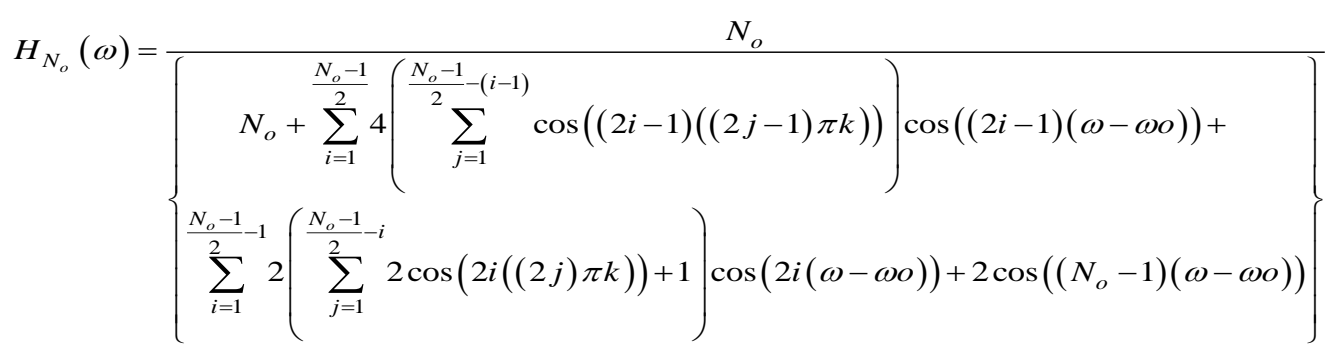

where $N_{o}$ is the odd number of samples in reference LFM.

Summarized flow chart for proposed filter which is designed to reduced sidelobe levels presented after matching filtering of LFM signals as shown in Figure 2.

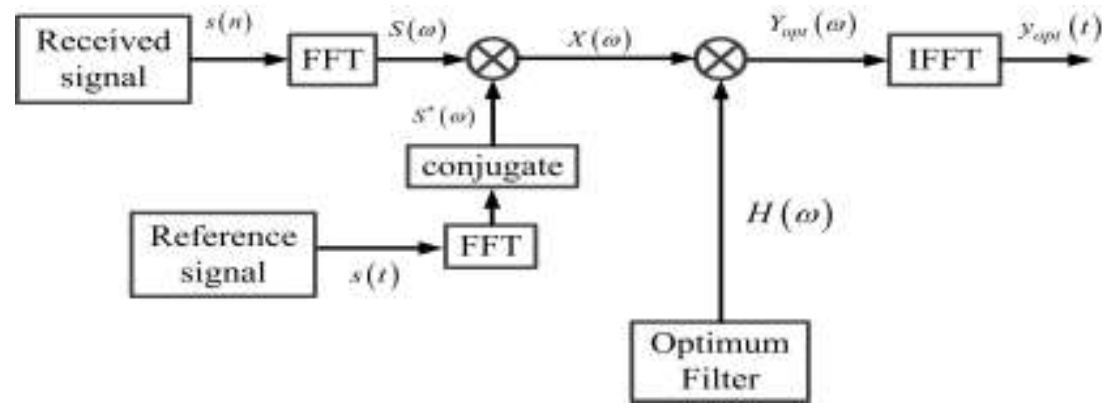

Figure 2. Optimum filter for sidelobe reduction 


\subsection{Even number samples}

The proposed optimum filter transfer function for even number of samples $(N=6)$ can be simplified as:

$$
H_{6}(\omega)=\frac{6}{\left\{\begin{array}{l}
6+2[1+2 \cos (2 k \pi)+2 \cos (4 k \pi)] \cos \left(k \pi+\omega-\omega_{o}\right)+ \\
4[\cos (2 k \pi)+\cos (6 k \pi)] \cos \left(2\left(k \pi+\omega-\omega_{o}\right)\right)+ \\
2[1+2 \cos (6 k \pi)] \cos \left(3\left(k \pi+\omega-\omega_{o}\right)\right)+ \\
4 \cos (4 k \pi) \cos \left(4\left(k \pi+\omega-\omega_{o}\right)\right)+2 \cos \left(5\left(k \pi+\omega-\omega_{o}\right)\right)
\end{array}\right\}}
$$

and for 8 samples, the transfer function can be obtained by:

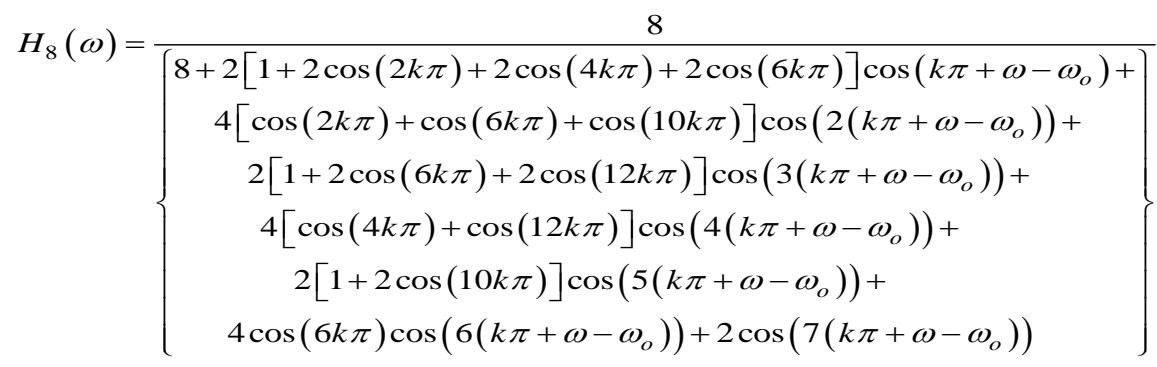

Hence, the transfer function in its general form for even samples $(N)$ can be expressed from (21) and (22) as:

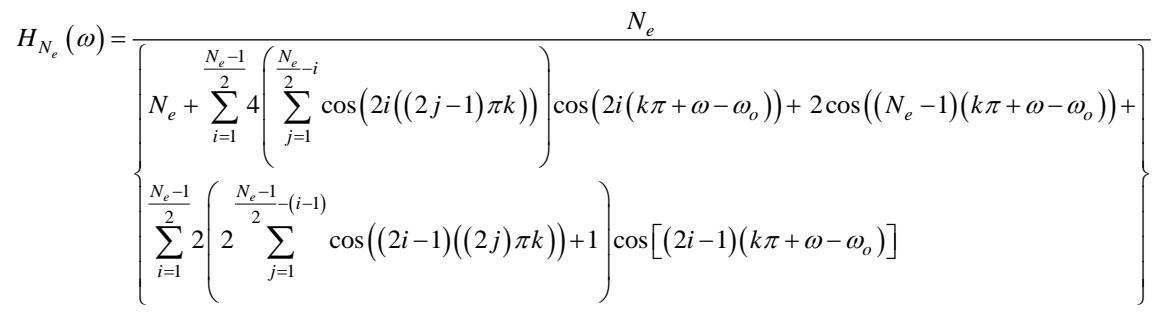

where $N_{e}$ is the even number of samples in reference.

\section{Discussing the ambiguity function in the LFM matched filtering and optimum filter}

SAR resolution strong depend on the Doppler return of targets so we can clarify the relation between time delay and Doppler frequency variation by calculating the ambiguity function for a given code and the corresponding cuts along zero delay and zero Doppler provide a strong indication about the code's characteristics in range and Doppler. Ambiguity function given by [1]

$$
\left|\chi\left(\tau ; f_{d}\right)\right|^{2}=\int\left|X^{*}(f) X\left(f-f_{d}\right) e^{-j 2 \pi f \tau} d f\right|^{2}
$$

where $\tau$ is the time delay, $f_{d}$ is the Doppler frequency, $\chi\left(\tau ; f_{d}\right)$ is the ambiguity function, and $\mathrm{X}(\mathrm{f})$ is the Fourier transform of the signal $\mathrm{x}(\mathrm{t})$. Three-dimensional plots for ambiguity function LFM matched filter are shown in figure 3(a) it is clear that sidelobes are exists with change in time delay and Doppler frequency. On the other hand, the 
sidelobes reduces successively in figure 3(b) which show the ambiguity function for optimum filter with change in time delay and Doppler frequency. Therefore, focusing on PSLR change with Doppler frequency variation which is reduced for optimum filter algorithm to $(-50 \mathrm{~dB})$ and decays with closing to zero Doppler frequency and reaches the same value after passing zero Doppler frequency rather than using LFM matched filtering which gives $(-13.2 \mathrm{~dB})$ constantly for all values of Doppler frequency as shown in figure (4)

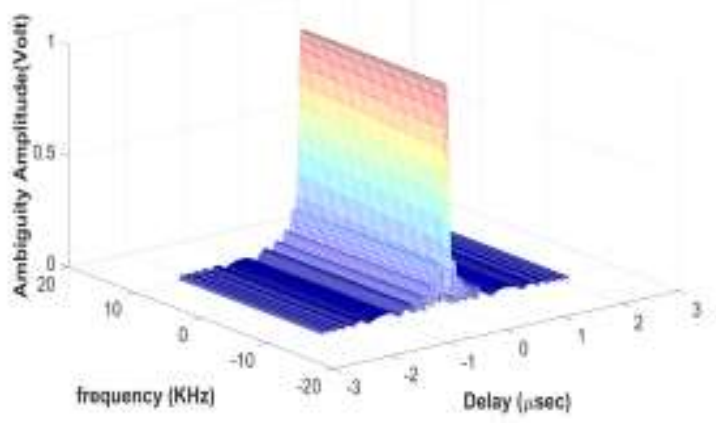

(a)

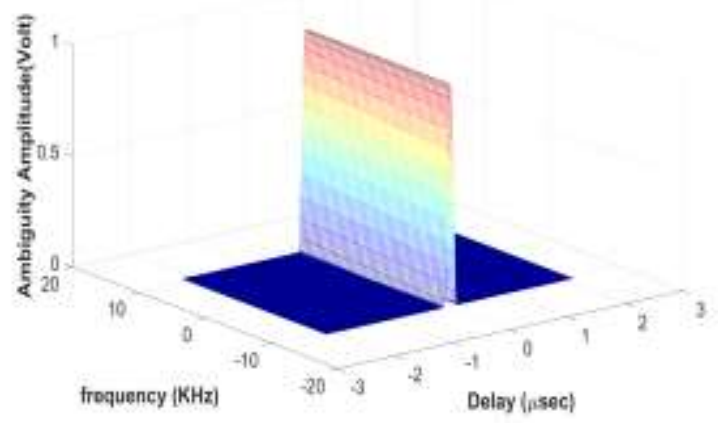

(b)

Figure 3. 3D Plot of ambiguity for (a)output of LFM matched filter (b) optimum filter

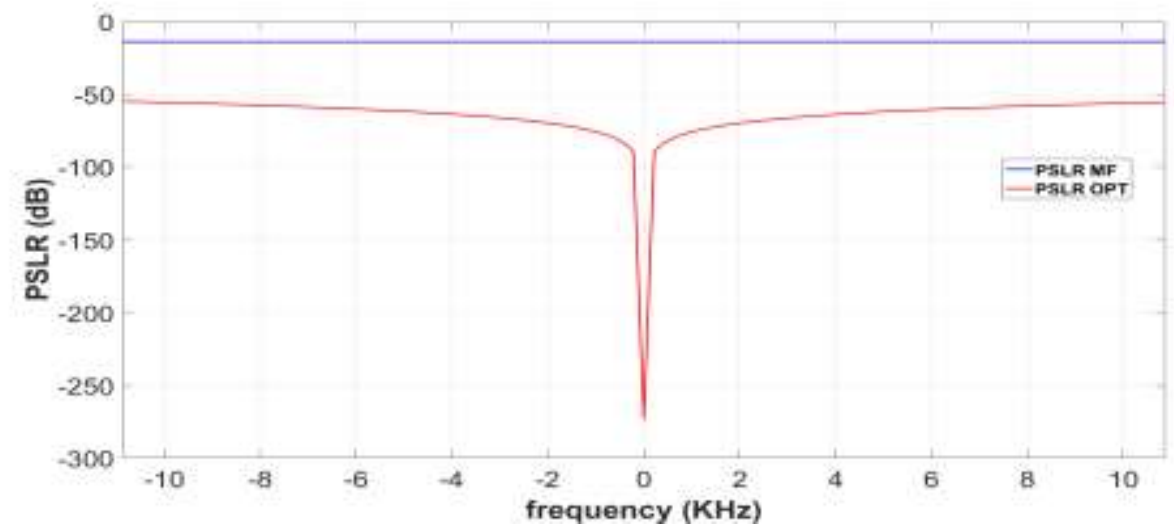

Figure 4. PSLR of LFM MF and OPT filter

\section{Proposed Sar Algorithm Flow Chart And Simulation Results}

As shown in figure (5) flow charts (a) Range Doppler algorithm (b) Proposed algorithm using optimum filter. Generation of raw data is done with the block diagram shown in figure (6) of airborne-SAR simulation. First input radar parameters of SAR that contains, carrier frequency $\left(f_{c}\right)$, chirp pulse duration $\left(T_{p}\right)$,chirp bandwidth $\left(B_{r}\right)$, sampling frequency $\left(f_{s}\right)$, pulse Repetition Frequency $(P R F)$, the azimuth antenna dimension $\left(L_{a}\right)$, range antenna dimension $\left(L_{r}\right)$, platform velocity $(V)$, and nominal height $(H)$. 


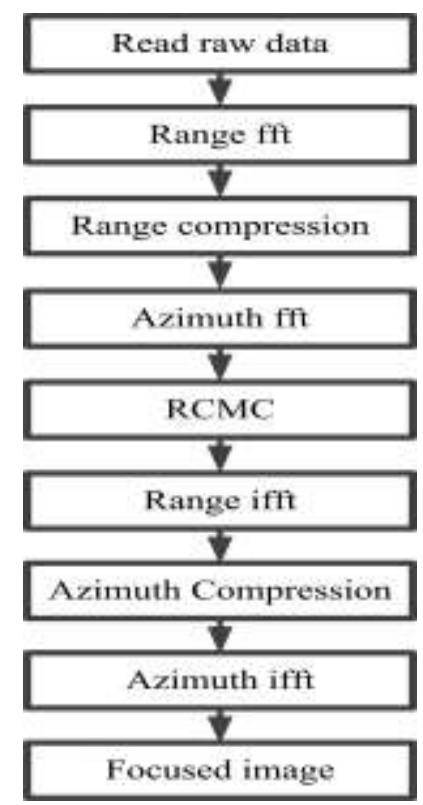

(a)

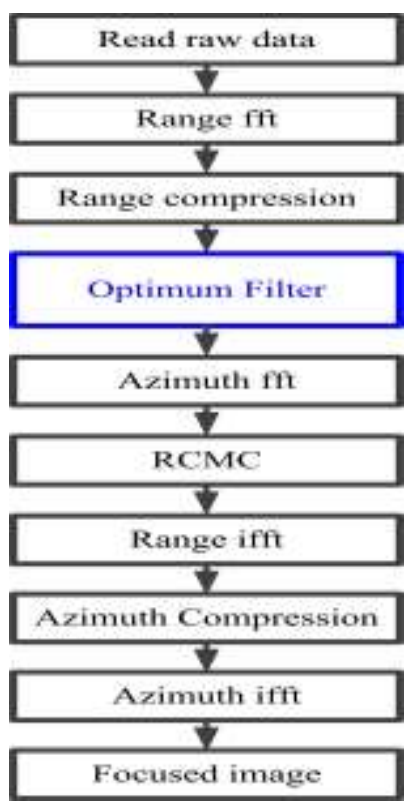

(b)

Figure5. SAR focused image algorithm (a) Range Doppler algorithm (b) Proposed algorithm

The computation radar parameters of SAR that contains, the wavelength $\left(\lambda=c / f_{c}\right)$, the chirp rate $\left(k_{r}=B_{r} / T_{p}\right)$, the beamwidth in azimuth $\left(\theta_{a}=\lambda / L_{a}\right)$, the beamwidth in range $\left(\theta_{r}=\lambda / L_{r}\right)$, the range pixel dimension $\left(R_{s}=c / 2 / f_{s}\right)$, the azimuth pixel dimension $\left(A_{s}=V / P R F\right)$, the range center of illuminated $\left(R_{c}=\sqrt{x_{c}^{2}+H^{2}}\right.$ where $x_{c}$ is the center of $\mathrm{X}$ axis of illuminated area), and the range swath $\left(W_{R}=R_{c} \theta_{r}\right)$.[9-11].

From the computation radar parameters and the target location used for computational illumination area that contains start and end position in azimuth, near and far range, number of azimuth samples $\left(N_{a}\right)$ of number of range samples $\left(N_{r}\right)$. Input nominal velocity, velocity error, and initial position (latitude, longitude, and altitude). Hence, velocity integration is used to obtain the position vector, which is in curvilinear coordinates (latitude, longitude, and altitude) as presented in the following [10].

$$
\left(\begin{array}{c}
\text { Lat }^{\prime} \\
\text { Long }^{\prime} \\
H^{\prime}
\end{array}\right)=\left(\begin{array}{ccc}
1 /(M+h) & 0 & 0 \\
0 & 1 /((N+h) \cos (L a t)) & 0 \\
0 & 0 & 1
\end{array}\right)\left(\begin{array}{l}
V x \\
V y \\
V z
\end{array}\right)
$$

where $V x, V y$ and $V z$ are the velocities at the east, north, and up coordinates, respectively, $M$ and $N$ are given by

$$
M=r_{e}\left(1-E^{2}\right) /\left(1-E^{2} \sin ^{2}(L a t)\right)^{3 / 2} \quad \text { and } \quad N=r_{e} /\left(1-E^{2} \sin ^{2}(L a t)\right)^{1 / 2}
$$

where $r_{e}=6378137 \mathrm{~m}$ is the equatorial radius, and $E^{2}=0.0818191908426$ is the first eccentricity. A typical X-band airborne SAR system is considered, the main parameters 
of which are listed in Table 1.

Table 1 Simulated Sensor Parameters

\begin{tabular}{|l|l|l|l|}
\hline Nominal height $(H)$ & $\mathbf{1 4 0 0 ~} \mathbf{~}$ & Range pixel dimension $\left(R_{s}=c / 2 / f_{s}\right)$ & $60 \mathrm{~cm}$ \\
\hline Slant range & $5504.8: 7961.8$ meter & Azimuth pixel dimension $\left(A_{s}=V / P R F\right)$ & $15 \mathrm{~cm}$ \\
\hline Wavelength $(\lambda)$ & $3 \mathrm{~cm}$ & Chirp duration $\left(T_{p}\right)$ & $5 \mu \mathrm{s}$ \\
\hline Platform velocity $(V)$ & $150 \mathrm{~m} / \mathrm{s}$ & Number of azimuth samples $\left(N_{a}\right)$ & 4096 \\
\hline $\begin{array}{l}\text { Pulse Repetition } \\
\text { Frequency }(P R F)\end{array}$ & $1000 \mathrm{~Hz}$ & Number of range samples $\left(N_{r}\right)$ & 4096 \\
\hline Sampling Frequency $\left(f_{s}\right)$ & $250 \mathrm{MHz}$ & Chirp bandwidth $\left(B_{r}\right)$ & $200 \mathrm{MHz}$ \\
\hline
\end{tabular}

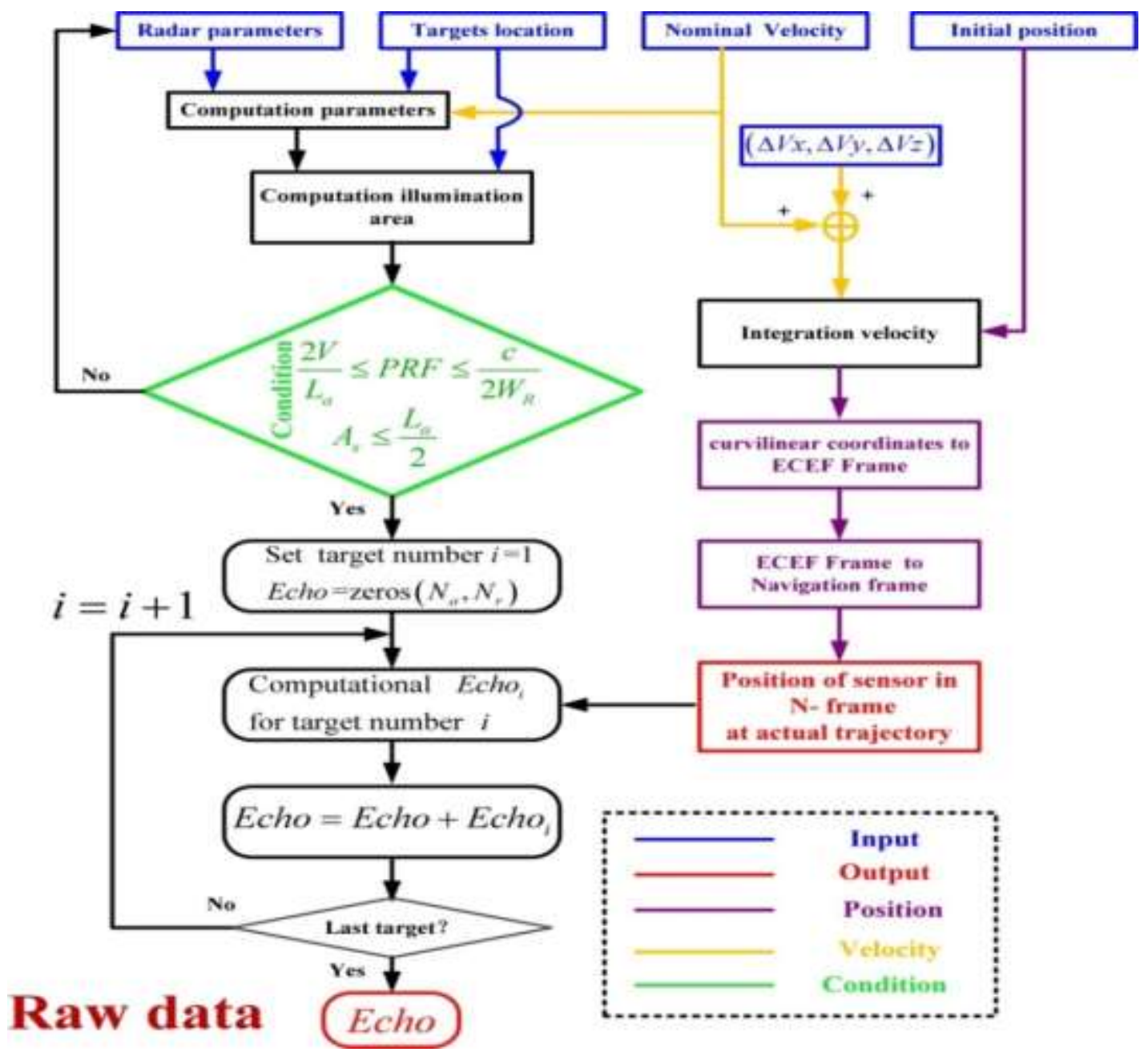

Figure 6. Generation of raw data

As shown above after generating the raw data then applying it to the proposed algorithm and compare the result with the traditional algorithm with matched filtering will 
result as shown in figure 7. First step is to generate the transmitted LFM signal as shown in figure 7 (a) which indicates in-phase (I) and quadrature phase $(\mathrm{Q})$ components

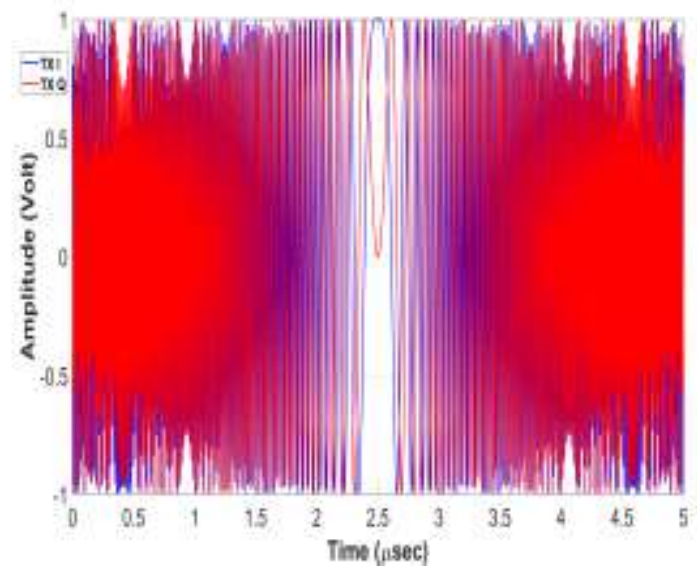

(a)

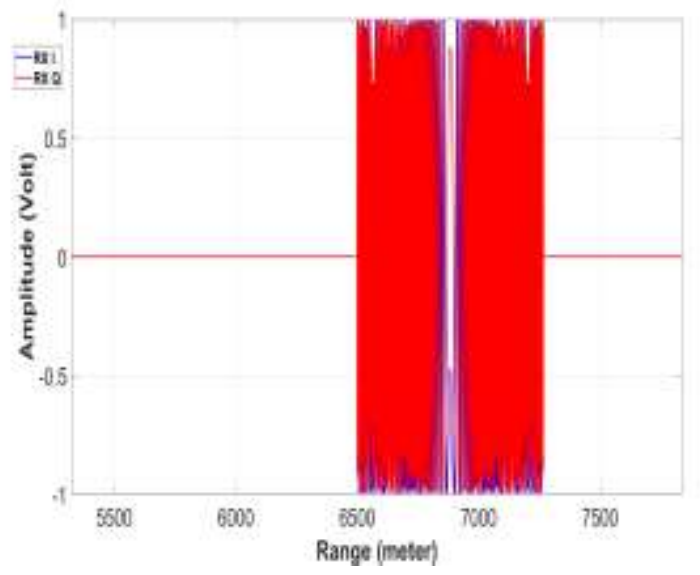

(b)

Figure (7). (a) LFM transmitted signal represented in-phase (I) and quadrature phase

(Q). (b) Target received signal at range (6500) meter represented with I and Q

The received simulated target position at range of (6500) meter so the amplitude of the signal as shown in figure 7 (b).

After this we have the received raw data acquired by aircraft of single target at range (6500) meter and zero azimuth position is shown in figure 8.

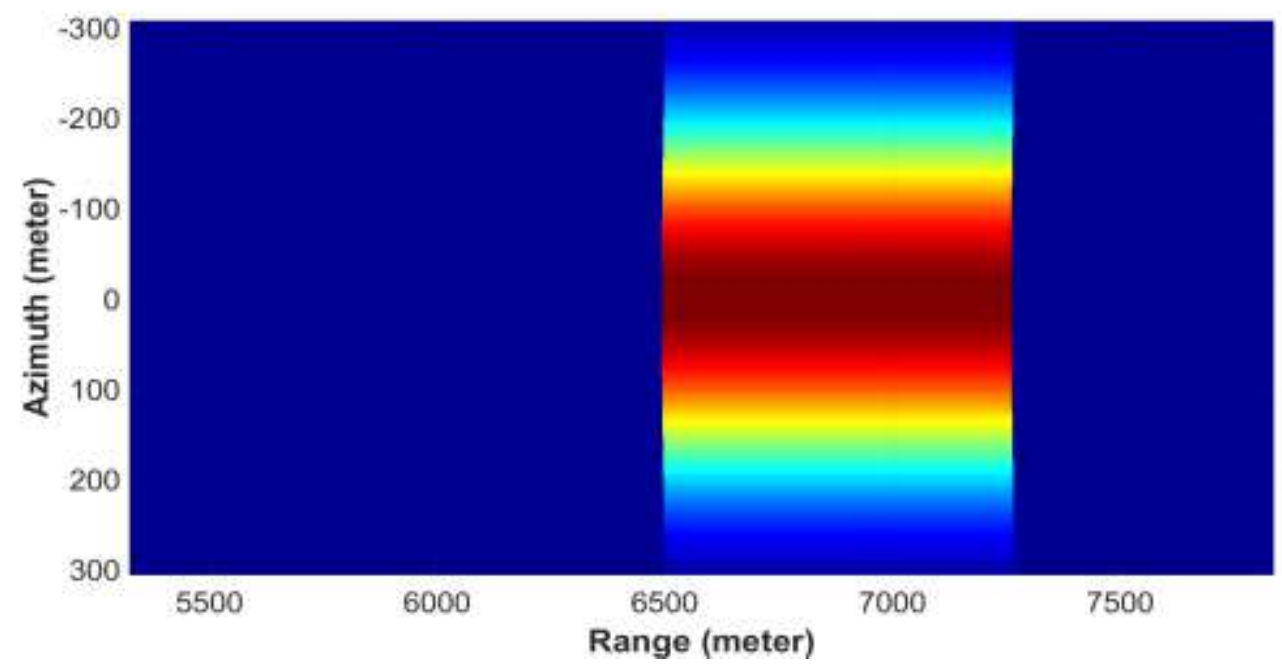

Figure 8. Image simulation of raw data received before processing

After this we will proceed to do the steps of our algorithm in parallel with the range Doppler algorithm, we notice that when using range Doppler algorithm, the sidelobes in range exists as shown in figure 9(a) and when using our proposed algorithm after range compression and optimum filter as shown in figure $9(\mathrm{~b})$ it reduces the side lobes in range 


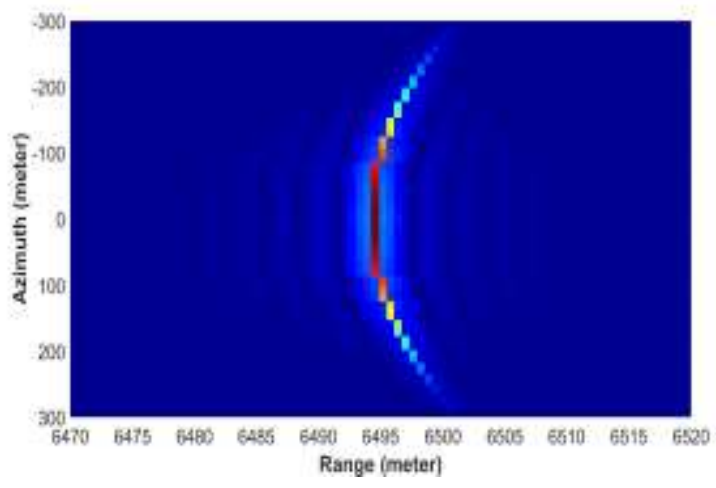

(a)

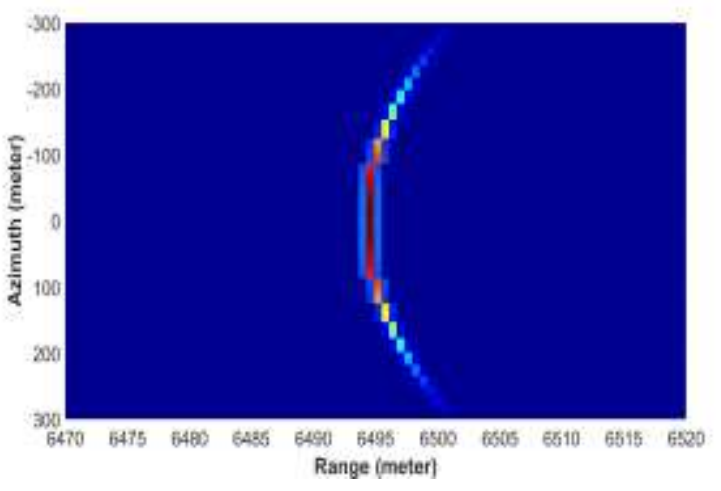

(b)

Figure 9. Range compression (a) RC for range Doppler (b) $R C$ for proposed algorithm

Taking a cross section of the image in data after range compression is shown in figure 10 the amplitude response of the matched filter colored blue and the output of optimum filter is colored as red it's obvious that the sidelobes is reduced in range when using the proposed algorithm with optimum filter.

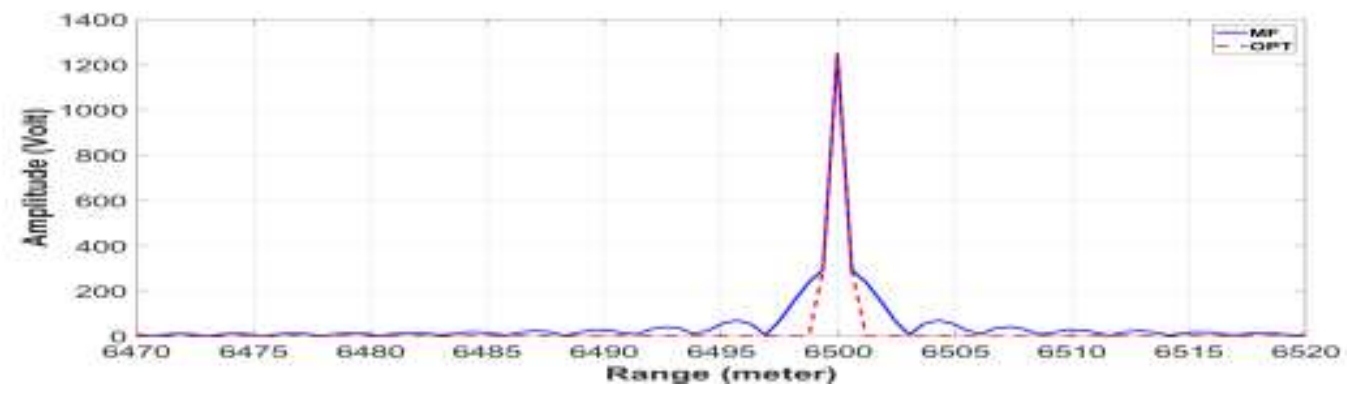

Figure 10. Amplitude response of output range compression with LFM matched filter and optimum filter

After RCMC it is also obvious that the sidelobes in range exists when using range Doppler algorithm as shown in figure 11(a) and sidelobes in range is reduced as shown in figure 11(b)

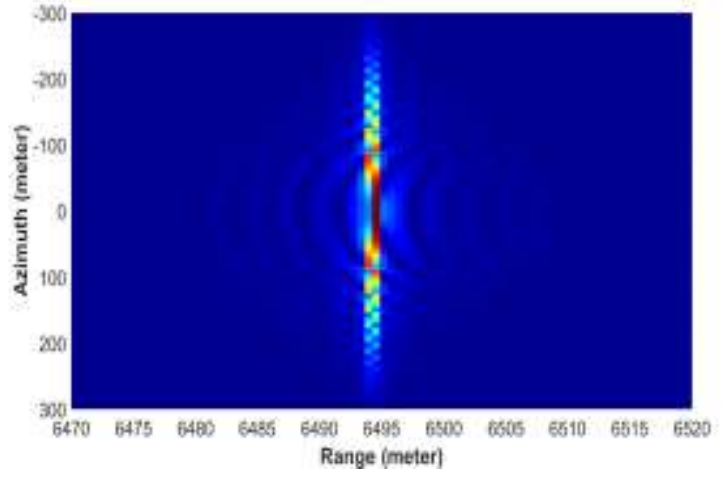

(a)

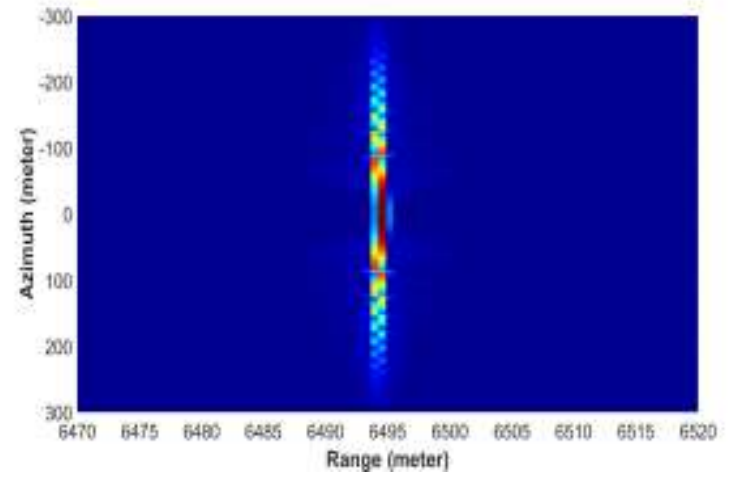

(b)

Figure 11. RCMC (a) using range Doppler algorithm (b) using proposed algorithm 
After azimuth compression it is also clear that that the sidelobes in range exists when using range Doppler algorithm as shown in figure 12(a) and sidelobes in range is reduced as shown in figure $12(b)$.

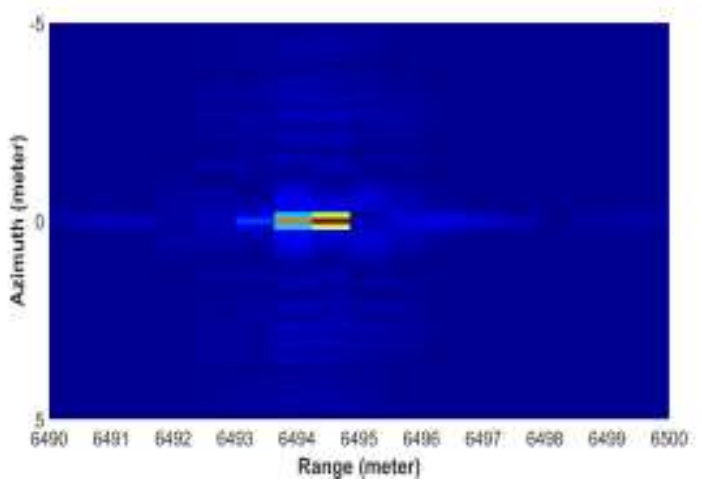

(a)

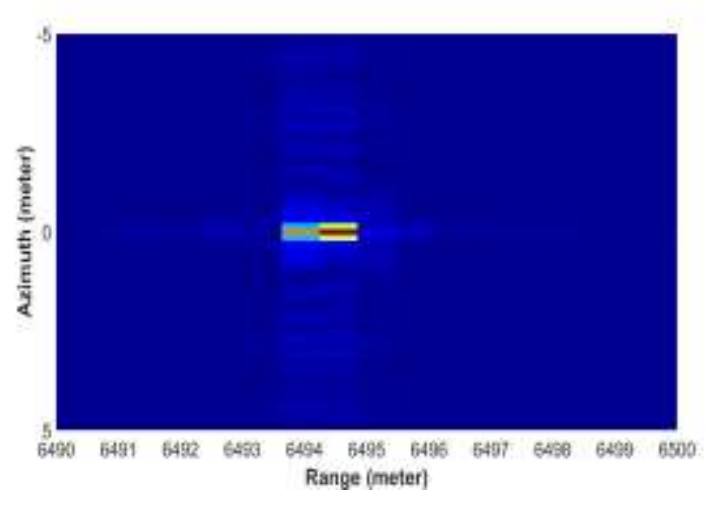

(b)

Figure 12. Azimuth compression (a) Range Doppler algorithm (b) Proposed algorithm

To clarify the enhancement, we take a cross section in amplitude response in range direction for is shown in figure 13(a) and amplitude response in azimuth is shown in figure 13(b) the range Doppler algorithm is represented by color blue and the proposed algorithm is represented by color red. The proposed algorithms reduce sidelobe level in range and azimuth directions.

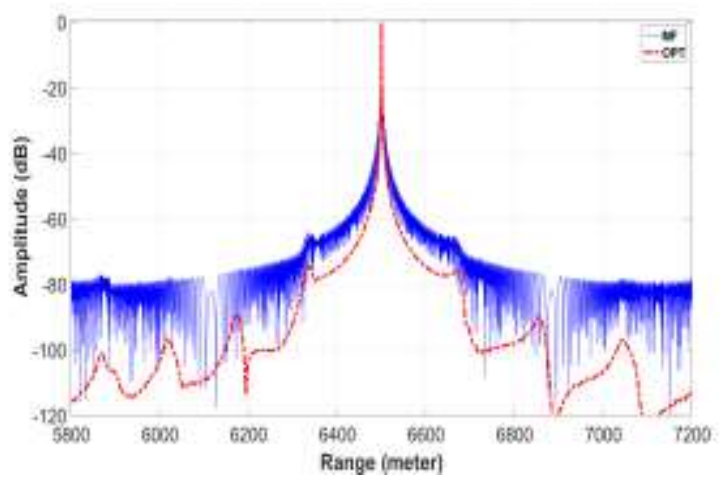

(a)

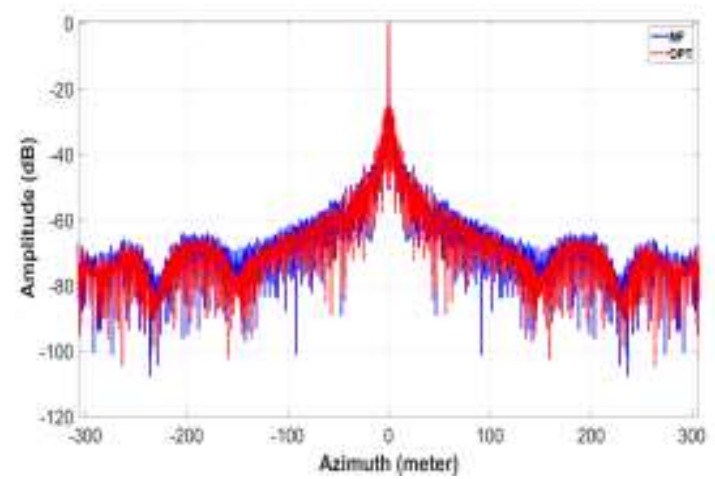

(b)

Figure 13. Amplitude response:(a) Range direction and (b) Azimuth direction

So from the previous simulation it is clear that using the proposed algorithm with optimum filter has an advantage of reducing of sidelobes much better than the range Doppler algorithm using matched filtering while keeping good range resolution.

\section{Implementation of proposed algorithm on real raw data comparing result with range Doppler algorithm}

The raw radar data used with an imaging resolution of $0.5 \times 0.5 \mathrm{~m}^{2}$ are collected by an experimental UAV-SAR, which was developed by the research institute of electronics 
technology in China [11] [12]. The experimental UAV-SAR system operated in X-band with bandwidth $300 \mathrm{MHz}$ The slant range of the scene center was $8 \mathrm{~km}$. The UAV flew at a height of about $5000 \mathrm{~m}$, at a speed of $110 \mathrm{~m} / \mathrm{s}$. It was not equipped with highly accurate inertial navigation units. The INS provided the motion measurements at the frequency of $40 \mathrm{~Hz}$, and it positioning accuracy was $1 \mathrm{~m} \mathrm{[11].}$

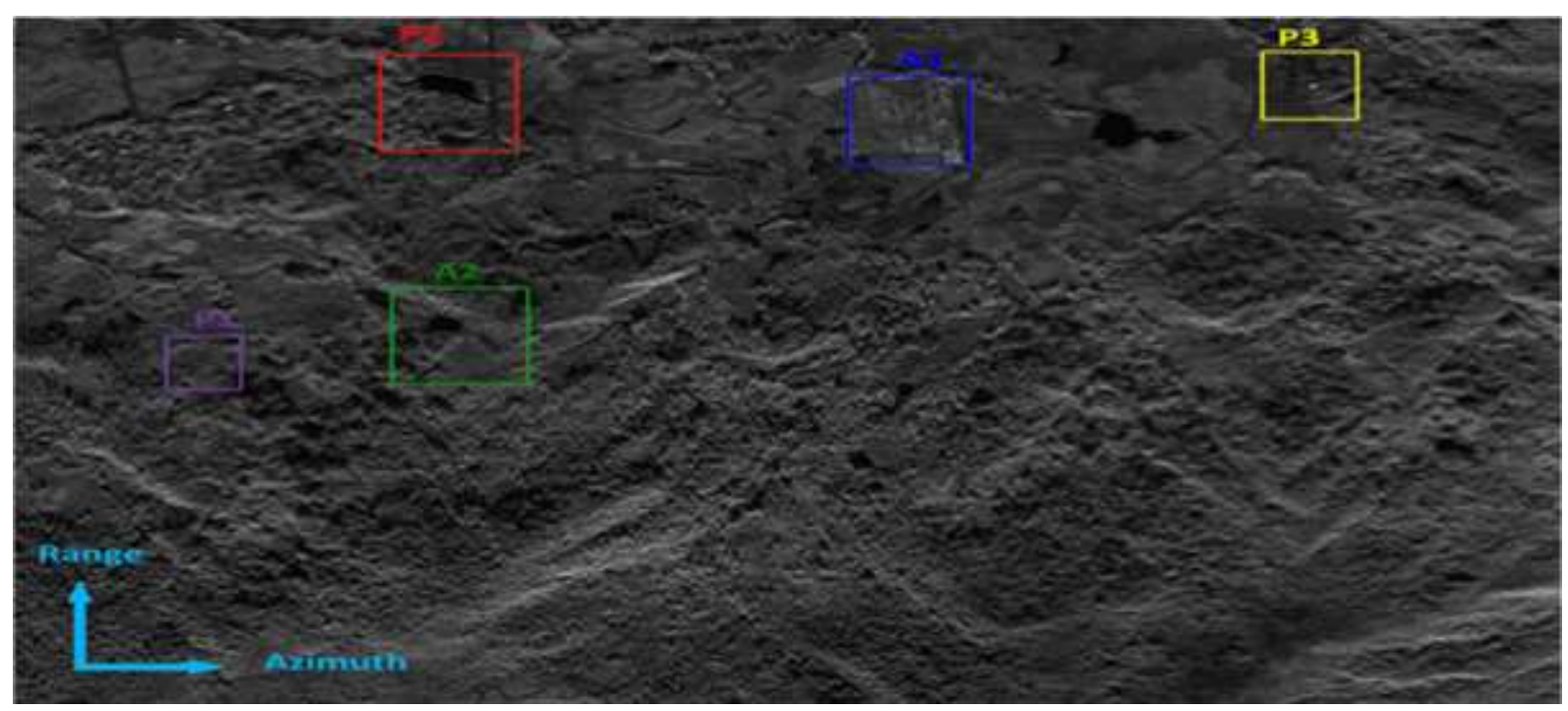

(a)

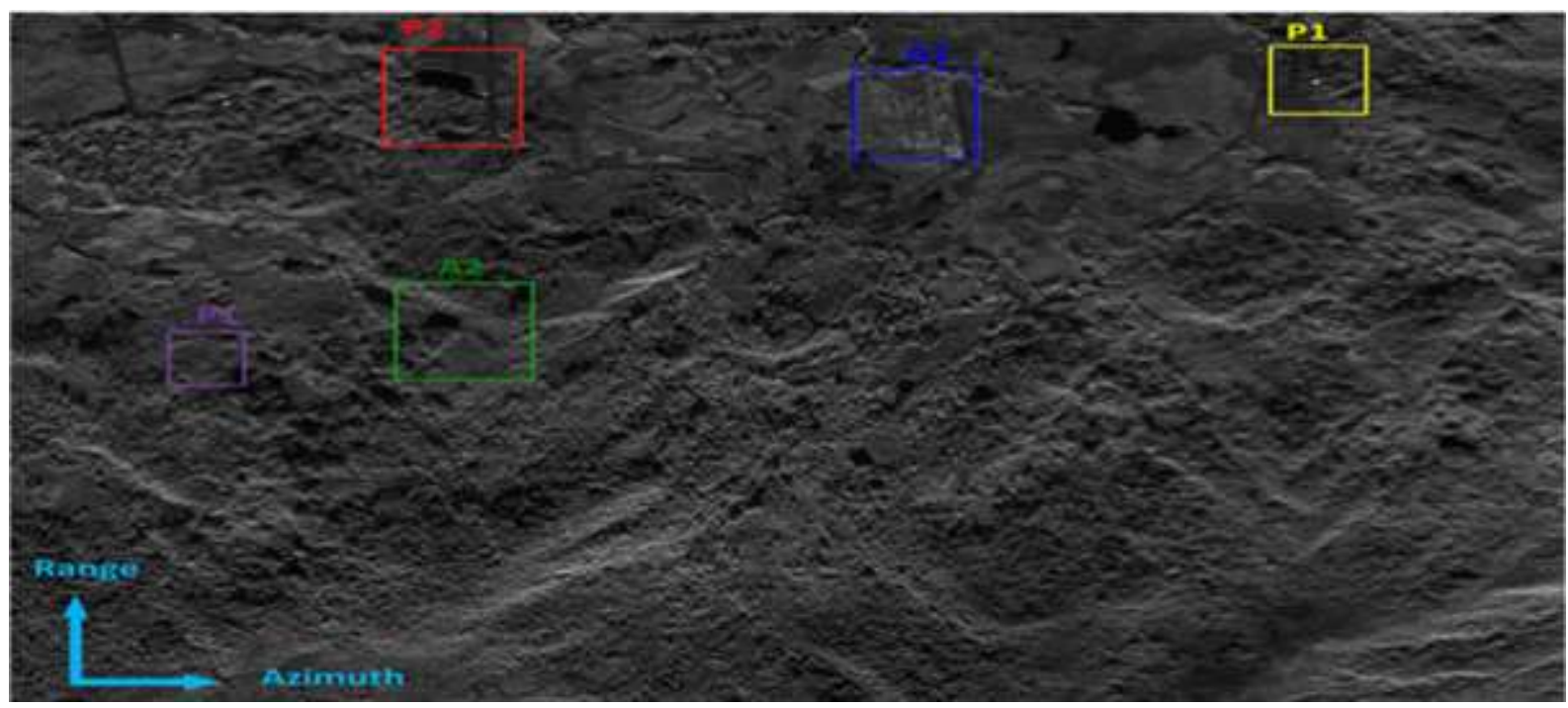

(b)

Figure 14. Resulted real image (a) Range Doppler algorithm (b) Proposed algorithm

Real raw data is applied on the range Doppler algorithm as shown in figure 14(a) and comparing the focused image with the proposed algorithm as shown in figure 14(b) results in better sidelobe reduction in range direction. As shown in figure (14) the highlighted squares (A1, A2) will be zoomed in and compared for range Doppler algorithm and the proposed algorithm to check for the enhancement achieved by the 
proposed algorithm as shown in figure 15 (a), (b). (c) and (d).

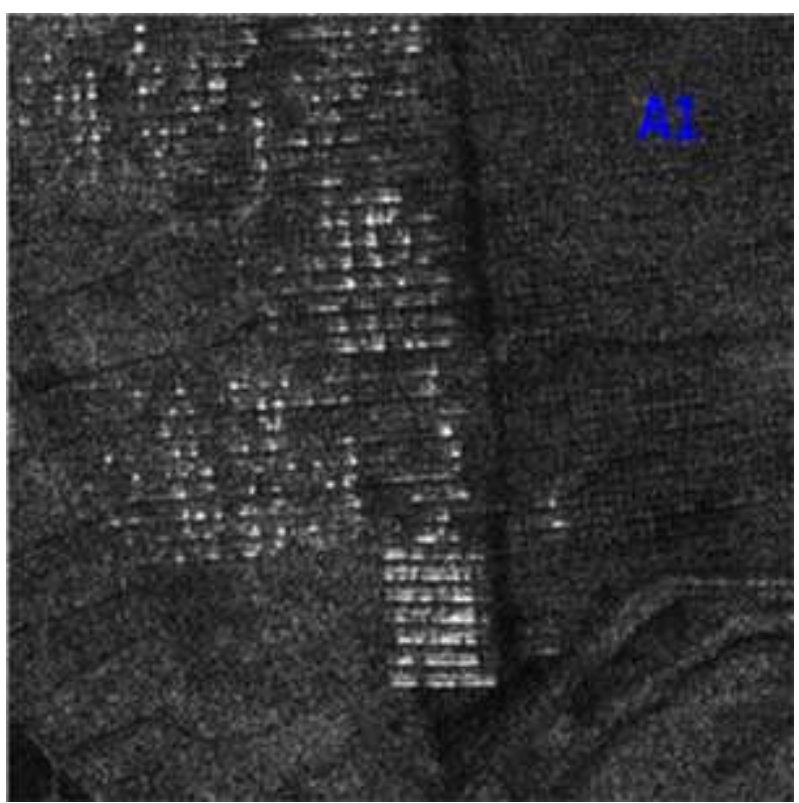

(a)

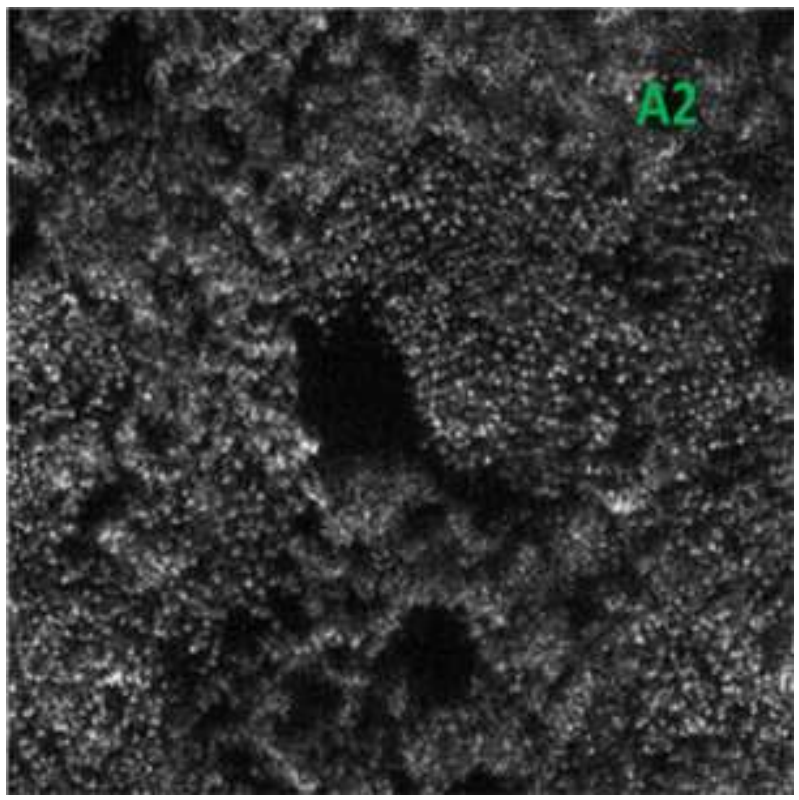

(c)

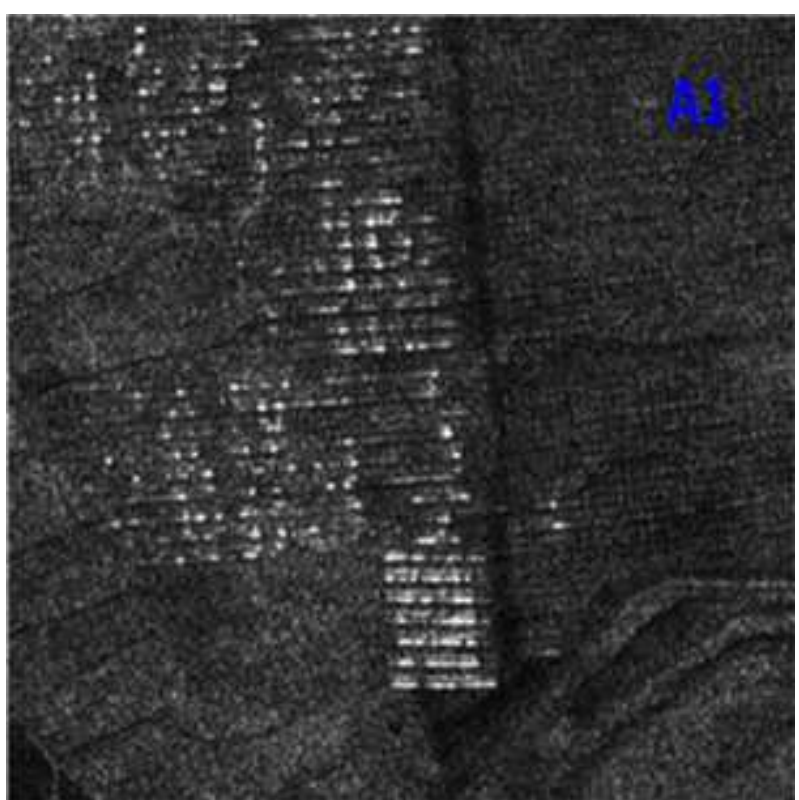

(b)

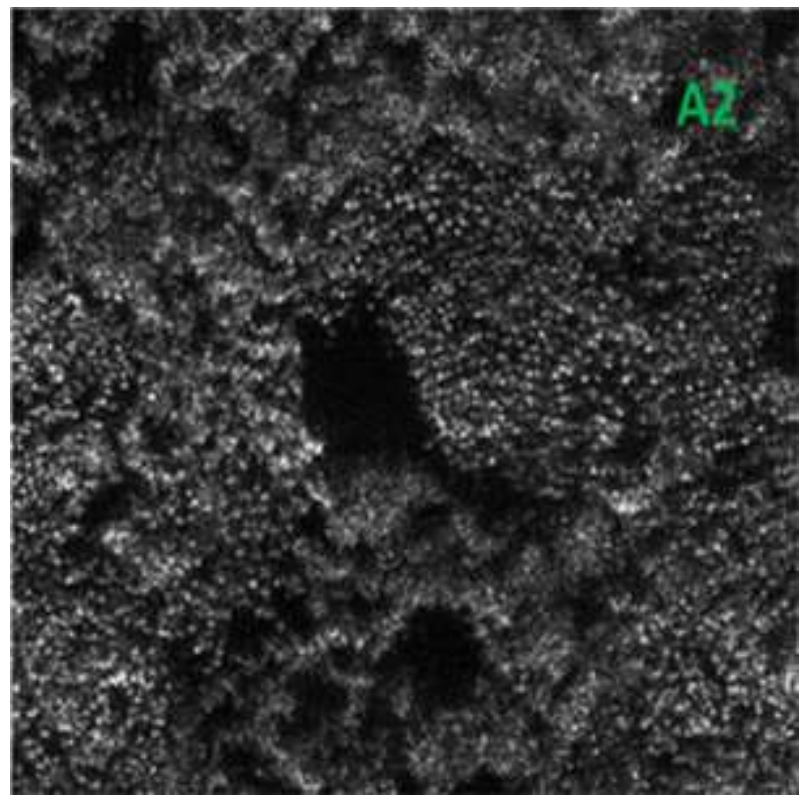

(d)

Figure 15. Chosen squares of resulted image: (a) and (c) range Doppler algorithm, (b) and (d) the proposed algorithm

For more clarification of the quality of the resulted image from proposed algorithm we choose tree areas with high reflection points as shown in figure 16 (a), (b), (c), (d), (e), and (f). Check the amplitude response of point's p1, p2 and p3 is shown in figure 17 (a), (b) and (c). 


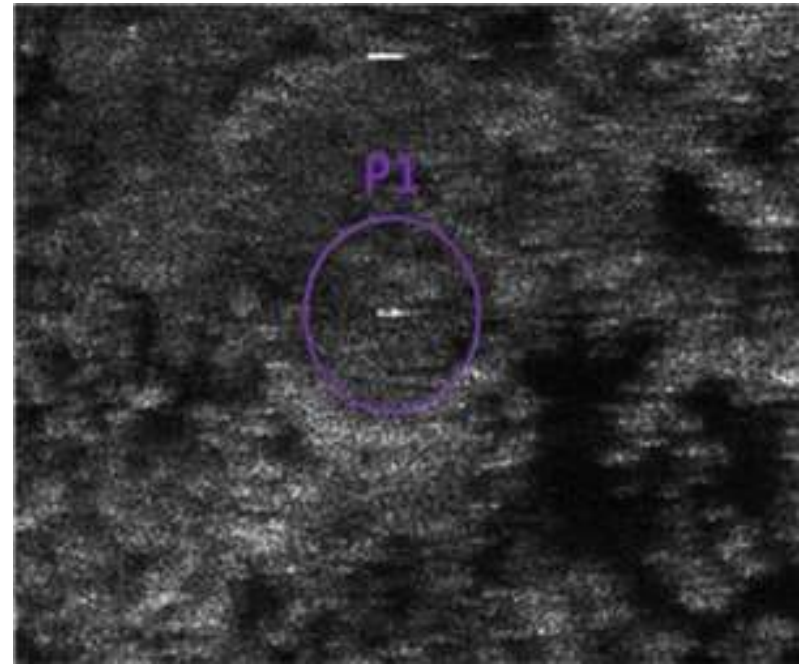

(a)

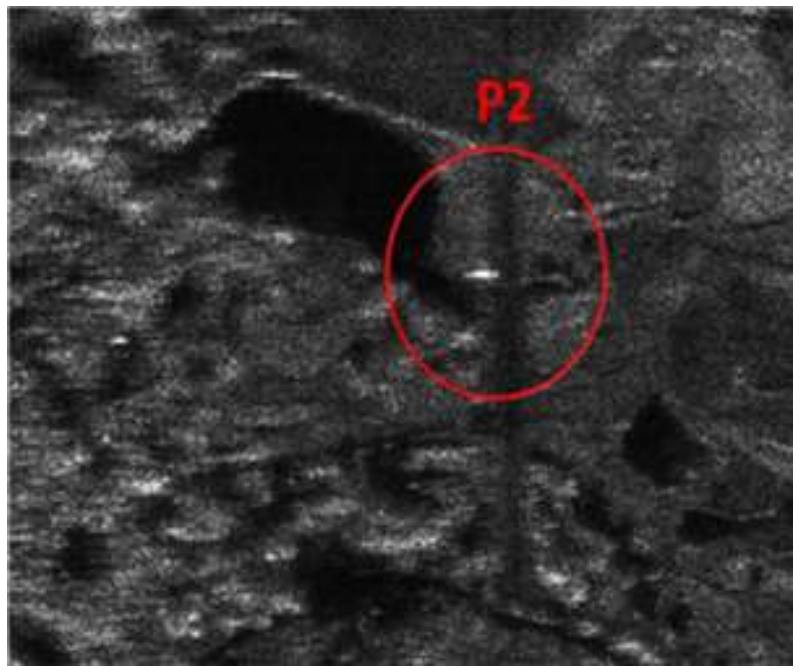

(c)

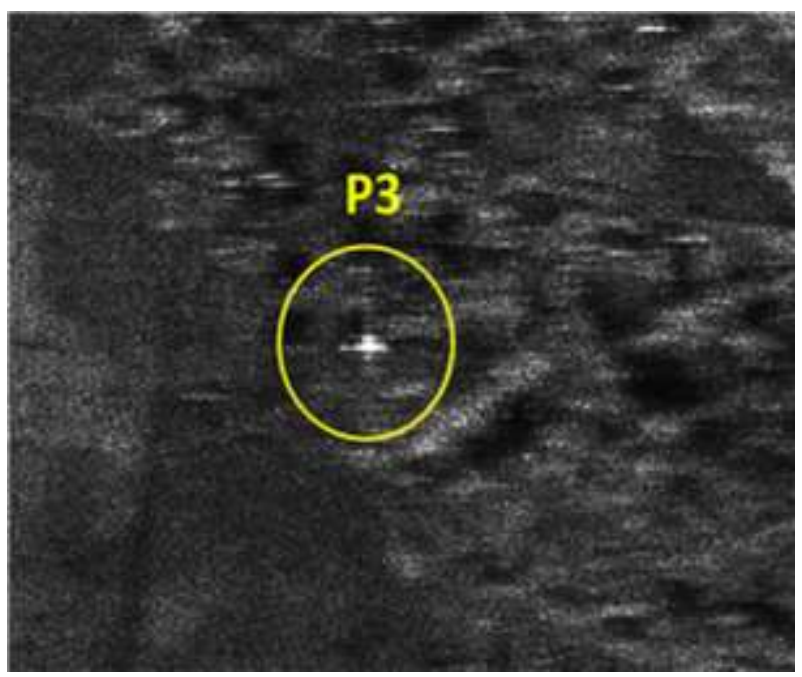

(e)

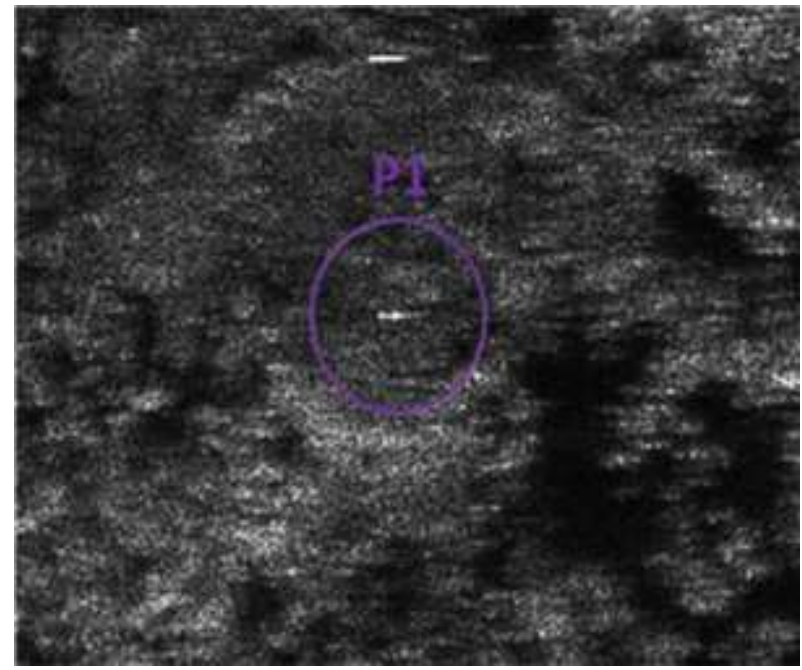

(b)

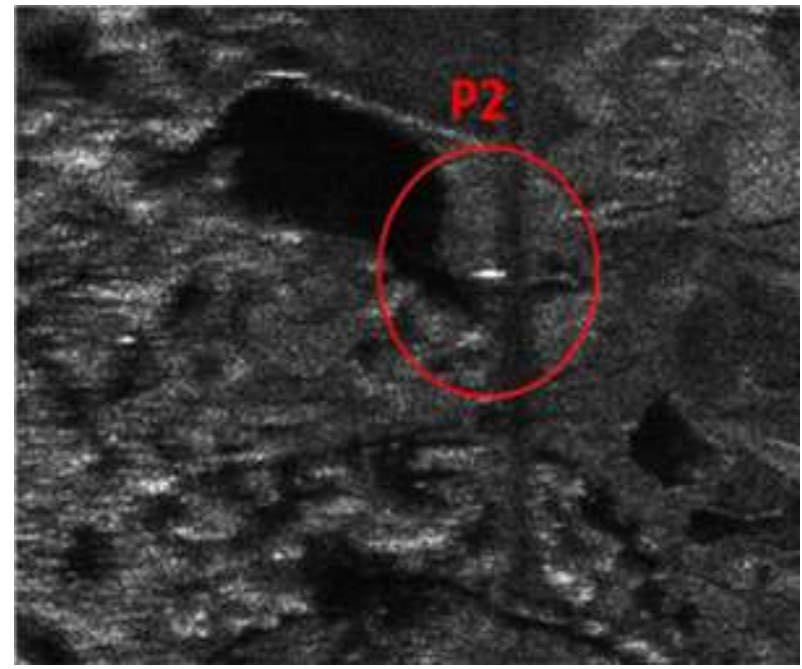

(d)

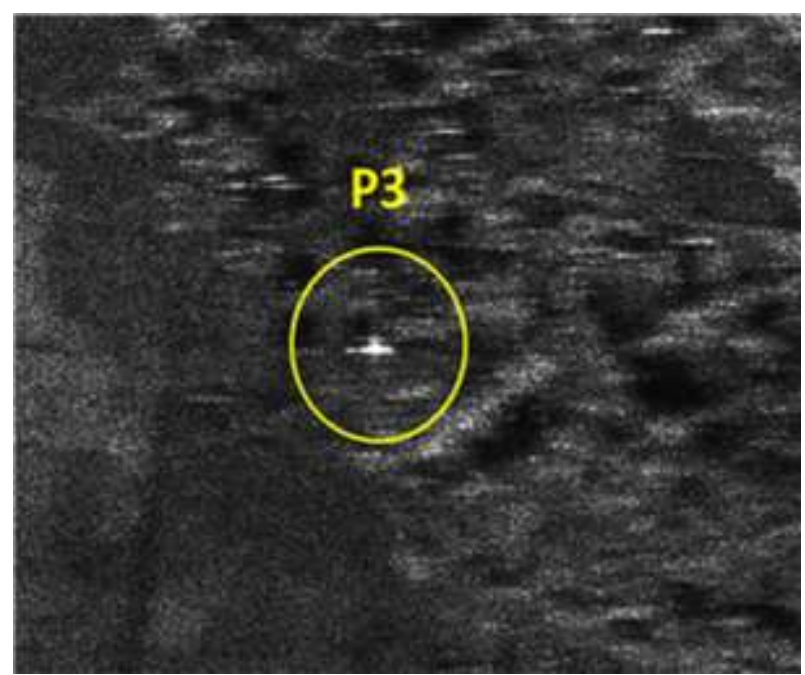

(f)

Figure 16. Chosen points for amplitude response with range comparison (a), (c), and (e) Range Doppler algorithm, (b), (d) and (f) the proposed algorithm 
As shown in figure 17 (a), (b) and (c), it is clear that the sidelobe amplitude level in range is higher using range Doppler algorithm which is represented by the color (blue) on the other hand it's reduced. The proposed algorithm represented by the color (red).

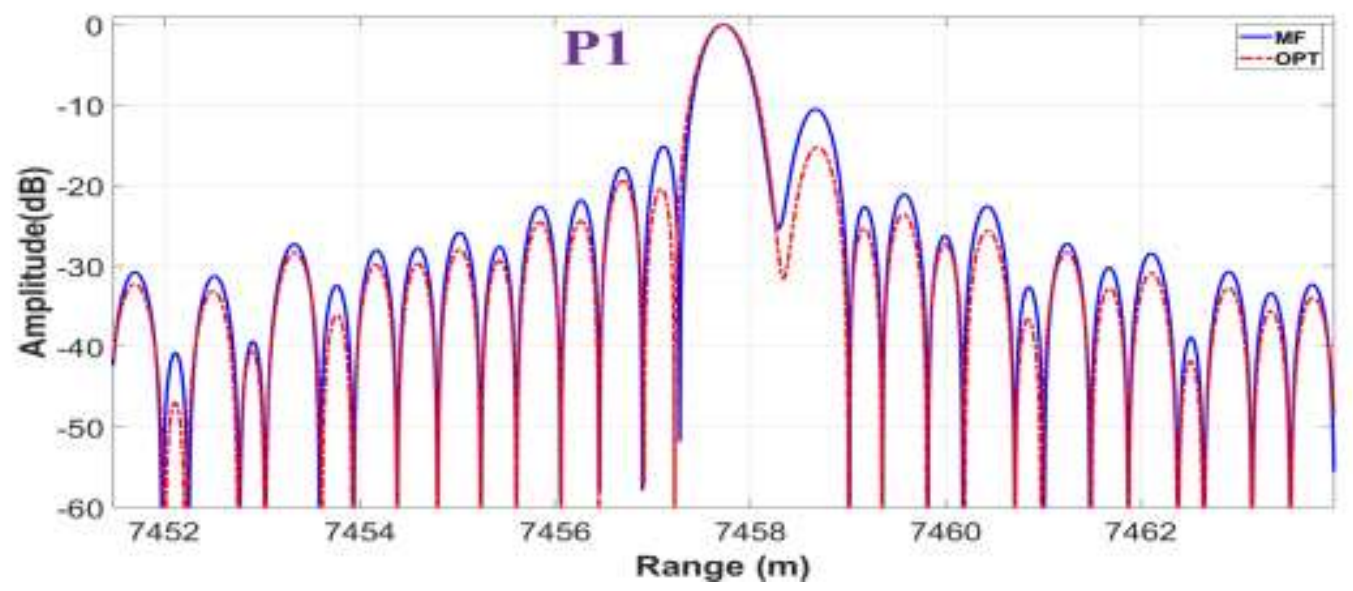

(a)

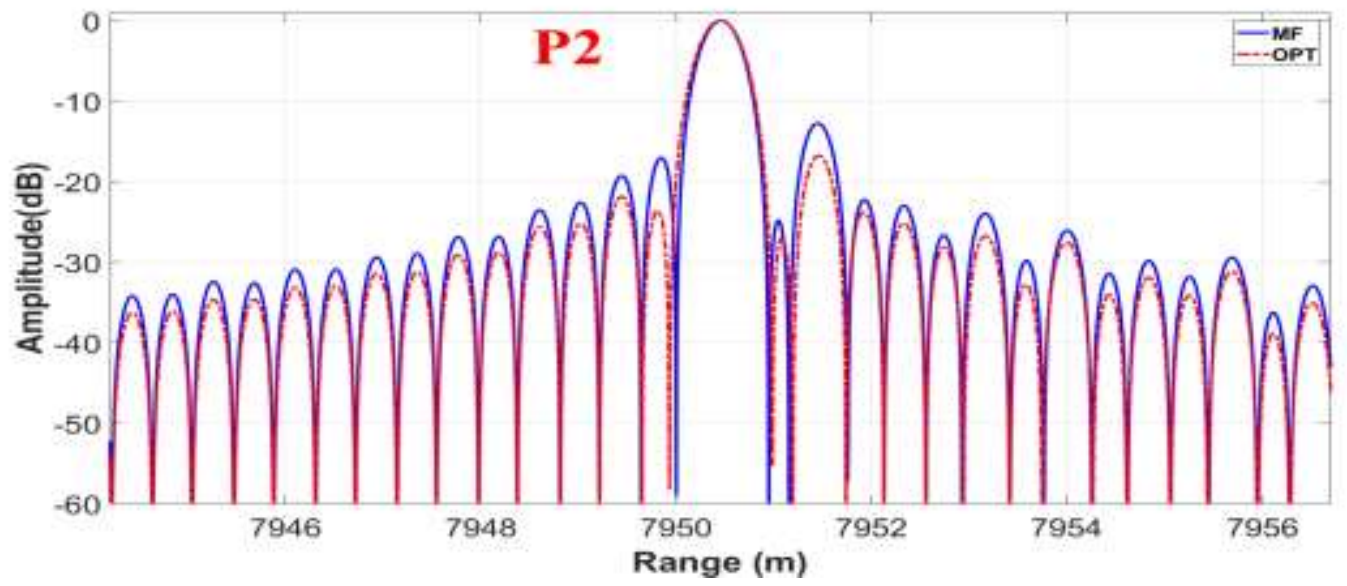

(b)

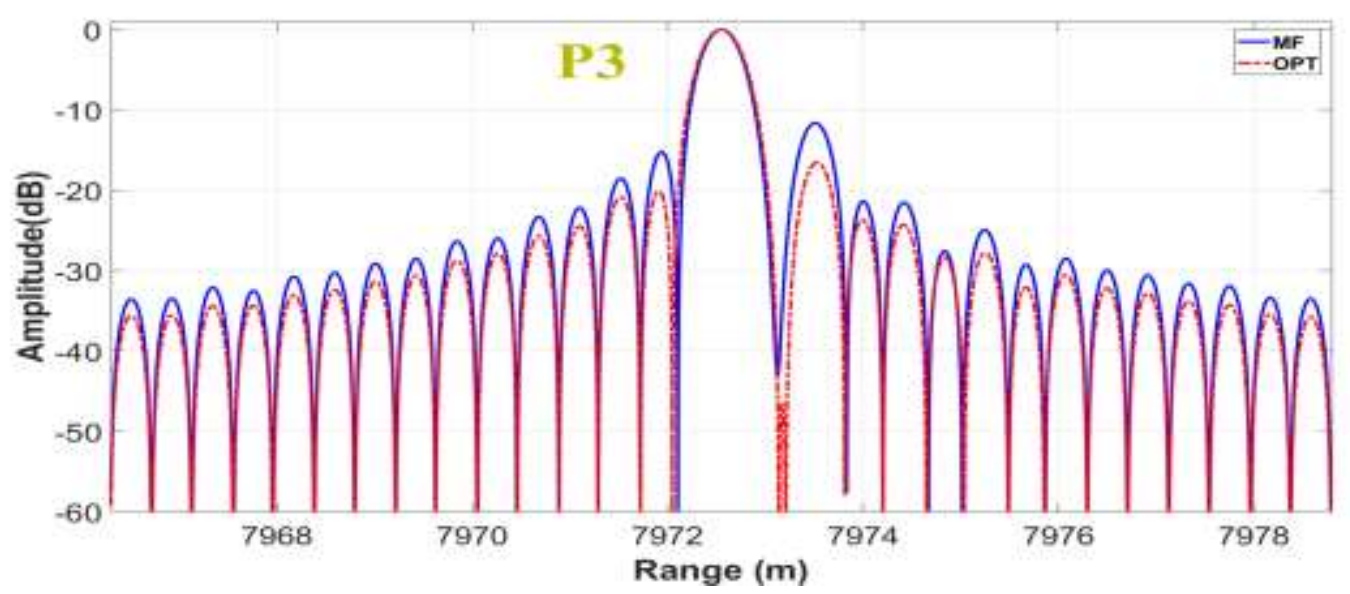

(c)

Figure 17. Amplitude response of chosen points with range variation (a) p1, (b) p2, and (c) p3 
To check the enhancement achieved by the proposed algorithm we need to calculate the entropy and the contrast of the resulted images from the proposed and range Doppler algorithms. The definition of the image contrast [9] can be expressed as follows:

$$
C o=\sqrt{E\left\{I^{2}(m, n)-E\left[I^{2}(m, n)\right]\right\}^{2}} / E\left[I^{2}(m, n)\right]
$$

where $C o$ stands for the image contrast, $I^{2}(m, n)$ stands for the pixel intensity of $(m, n)$, and $E[\bullet]$ stands for the mean value of the image intensity. The entropy of an image is defined as follows [7,9].

$$
\text { Ent } \left.=-\sum_{\text {Height Width }} \sum^{2}(m, n) / S\right) \ln \left(I^{2}(m, n) / S\right)
$$

where $S=\sum_{\text {Heigh }} \sum_{\text {Widh }} I^{2}(m, n)$ is the total energy of image. So we can obtain the following results of the obtained focused images by the proposed and range Doppler algorithms as shown in table (2)

Table (2) Obtained results

\begin{tabular}{|l|l|l|l|l|}
\hline & & Entropy & Contrast & Type of Algorithm \\
\hline Figure (17) & (a) & 15.6712 & 1.7802 & Range Doppler \\
& (b) & 15.6540 & 1.8079 & Proposed Algorithm \\
\hline & (a) & 11.0114 & 2.0356 & Range Doppler \\
\hline & (b) & 10.9941 & 2.0722 & Proposed Algorithm \\
& (c) & 10.9904 & 1.8000 & Range Doppler \\
& (d) & 10.9537 & 1.8473 & Proposed Algorithm \\
\hline & (a) & 11.1225 & 1.5573 & Range Doppler \\
& (b) & 11.1053 & 1.5978 & Proposed Algorithm \\
\hline Figure (19) & (c) & 11.5231 & 2.1278 & Range Doppler \\
& (d) & 11.5043 & 2.1647 & Proposed Algorithm \\
& (e) & 11.4114 & 8.3376 & Range Doppler \\
& (f) & 11.3926 & 8.4960 & Proposed Algorithm \\
\hline
\end{tabular}

As shown in table 2 our proposed algorithm enhances the contrast and the entropy levels compared to the range Doppler algorithm by reducing the sidelobe level so the small targets masked by the side lobes of large targets will appear enhancing the image quality.

\section{Conclusion and future work}

In this paper, a new algorithm for SAR image formation that reduced sidelobe in range direction. Applied the proposed algorithm to simulated SAR raw data the side loop reduced than the range Doppler algorithm. Finally, test of the proposed algorithm on real raw data of SAR comparing the focused image with the range Doppler algorithm shows that the focused image of the proposed algorithm has enhancement by measured the entropy decrease and contrast increase. Future work 
will be sidelobe reduction in azimuth direction using proposed algorithm with satellite raw data

\section{References}

[1] Mark A Richards,James A. Scheer,William A. Holm, Principles of modern radar "basic principle" Published by SciTech Publishing - An Imprint of the IET 379 Thornall Street Edison, NJ 08837 scitechpub.com

[2] lan G.Cumming, Frank H.Wong Digital Processing Of Synthetic Aperture Radar Data Algorithms And Implementation Published by ARTECH HOUSE, INC.685 Canton street Norwood,MA 02062

[3] Bu Chin Wang Book Wiley Interscience Digital Signal Processing Techniques and Applications in Radar Image Processing Aug 2008 eBook-DDU Published by John Wiley \& Sons, Inc . Hoboken, New Jersey Published simultaneously in Canada

[4] . R. Mahafza, Radar Systems Analysis and Design using Matlab, 3rd ed., RCR press, Alabama, USA, 2013.

[5] M. Archana and M. Gnana, "Low power LFM Pulse Compression Radar with Sidelobe Suppression," International Journal of Advanced Research in Electrical, Electronics and Instrumentation Engineering (IJAREEIE), Vol. 3, Issue 7, pp. 10627-10679, July 2014.

[6] M. I. Skolnik, Introduction to Radar Systems. 3rd ed., McGraw-Hill, New York, 2002.

[7] L. R. Varshney and D. Thomas,"Sidelobe Reduction for Matched Filter Range Processing," in Proceedings of the 2003 IEEE Radar Confererence, pp. 446451, May 2003.

[8] A. K. Sahoo and G. Panda, "Sidelobe reduction Of LFM signal using convolutional windows," International Journal of Signal and Imaging Systems Engineering, Vol. 5, Issue 4, pp. 253-259, 2012.

[9] Berizzi and G. Corsini, "Autofocusing of inverse synthetic aperture radar images using contrast optimization," IEEE Transactions on Aerospace and Electronic Systems, vol. 32, pp. 1185-1191, 1996

[10]J. Wang and X. Liu, "SAR Minimum-Entropy Autofocus Using an Adaptive-Order Polynomial Model," IEEE Geoscience and Remote Sensing Letters, vol. 3, pp. 512-516, 2006

[11]A. Azouz, and Z. Li, "Improved phase gradient autofocus algorithm based on segments of variable lengths and minimum entropy phase correction", IET Radar, Sonar \& Navigation ( Volume: 9, Issue: 4, 4 2015) Page(s): 467 - 479 F.

[12]A. Azouz and Z. Li, "Motion compensation for high-resolution automobile-SAR", International Journal of Remote Sensing, pp. 4815-4838, 2014/07/03 2014 